\title{
Olivier de Serres et son Théâtre d'agriculture
}

Olivier de Serres and his Théâtre d'agriculture

\section{Hélène Vérin}

\section{OpenEdition}

\section{Journals}

Édition électronique

URL : http://journals.openedition.org/artefact/405

DOI : $10.4000 /$ artefact.405

ISSN : 2606-9245

\section{Éditeur :}

Association Artefact. Techniques histoire et sciences humaines, Presses universitaires du Midi

\section{Édition imprimée}

Date de publication : 1 octobre 2016

Pagination : 161-180

ISBN : 978-2-7535-5174-9

ISSN : 2273-0753

Référence électronique

Hélène Vérin, "Olivier de Serres et son Théâtre d'agriculture », Artefact [En ligne], 4 | 2016, mis en ligne le 07 juillet 2017, consulté le 19 avril 2019. URL : http://journals.openedition.org/artefact/405 ; DOI : 10.4000/artefact.405 


\section{Olivier de Serres et son Théâtre d'agriculture}

Hélène VÉRIN"

\section{Résumé}

Olivier de Serres a ouvert la voie de la science agricole moderne et de la sériciculture en France. Son important ouvrage, Le Theatre d'agriculture et mesnage des champs, publié en 1600, appartient à une double tradition : celle des écrits sur l'agriculture et celle des écrits sur les arts de la Renaissance. Après une présentation de l'auteur, nous nous attacherons à inscrire cette œuvre dans le contexte de ces traditions. Une dernière partie sera centrée sur la littérature consacrée à l'élevage des vers à soie durant la Renaissance en Europe.

Mots-clés : expérience, invention, raison, réduire en art et méthode, science pratique.

\section{Abstract. Olivier de Serres and his Théâtre d'agriculture}

Olivier de Serres opened the way to modern agricultural science and sericulture in France. His important book, Le theatre d'agriculture et mesnage des champs, published in 1600, belongs to a double tradition: the writings on agriculture and the writings on arts, in the Renaissance. After a presentation of the author, we will try to study this work within the context of both traditions. A final section will focus on the literature dedicated to the breeding of silkworms during the Renaissance in Europe.

Keywords : experiment, invention, practical science, reason, to reduce in art and method.

\footnotetext{
*. Docteur d'État en philosophie, Hélène Vérin est chercheuse au CNRS en économie, philosophie puis histoire. Ses recherches portent sur l'histoire et la philosophie des techniques et des sciences de l'ingénieur à l'époque moderne. Elle a récemment publié en collaboration Réduire en art, la technologie de la Renaissance aux Lumières (Paris, MSH, 2008) et réédité Entrepreneurs, entreprise. Histoire d'une idée (Paris, Classiques Garnier, 2011). Contact [verin.helene@me.com].
} 
Le theatre d'agriculture et mesnage des champs d'Olivier de Serres, seigneur du Pradel, publié en 1600, ouvre la voie de la science agricole moderne ${ }^{1}$, telle est l'opinion généralement reçue en France. Suivant un modèle classique dans ce type de traité, Serres y campe le personnage $\mathrm{du}$ « bon mesnager » dirigeant un domaine en bon père de famille. La clarté de l'écriture, la richesse et la précision des observations, des descriptions, des opérations préconisées, une langue alerte et vigoureuse entraînent le lecteur à voir dans le domaine présenté celui du Pradel et dans son maître Olivier de Serres lui-même.

Après une présentation de l'auteur, nous nous attacherons à inscrire cette œuvre dans le contexte des écrits sur les arts de la seconde Renaissance et des modèles d'exposition qu'ils promeuvent.

\section{La personne, le personnage}

Personne, personnage, Olivier de Serres lui-même joue sur la confusion.

Fils d'un marchand drapier protestant, d'une famille de notables de Villeneuvede-Berg, dans le Vivarais, c'est en 1558, à l'âge de 19 ans, qu'il achète le Pradel, plus tard constitué en un vaste domaine de cent cinquante hectares. Il continue à habiter Villeneuve-de-Berg et assure le diaconat de l'église réformée de cette ville. En 1571, en acquérant les droits de haute, moyenne et basse justice sur son domaine, Serres prend le titre de seigneur du Pradel et, en 1578, il s'y installe. Dans les désordres épouvantables de cette époque, en tant que réformé et notable, il doit assurer sa part de responsabilités et il est impliqué, quoi qu'il fasse ou dise plus tard. Malheureusement, sur l'aménagement de sa vie et de son domaine jusqu'à l'année 1605, nous n'avons que peu de chose, mais nous avons trace d'un épisode assez épineux, celui du séjour de Jacques Besson, pasteur de Villeneuvede-Berg, de 1562 à 1564.

L'histoire de Besson croise en effet celle de Serres et ils ont en commun d'être tous deux auteurs de " théâtres ", Thêâtre des instruments et machines pour Besson, publié en 1578, Théâtre d'agriculture pour Serres.

Jacques Besson², ami du fameux naturaliste Conrad Gesner et bien introduit dans les milieux érudits de Genève, était connu et apprécié en Suisse comme ingénieur, mathématicien et distillateur de plantes médicinales. Cependant, en mars 1562, il doit quitter Genève pour Villeneuve-de-Berg où il a été appelé pour remplir les fonctions de pasteur. Ce départ s'inscrit dans un mouvement plus général de recrutement de pasteurs. Mais, que ce soit lui et pas un autre qu'Olivier de Serres demande et vient chercher à Genève suppose qu'il a été appelé à d'autres tâches que celles d'édification pastorale.

Pendant deux ans, Besson exerce son ministère, puis les choses se gâtent, comme il le raconte de Lyon, à la fin de l'année 1564, dans une lettre adressée à Nicholas Colladon, alors secrétaire de la Compagnie des Pasteurs de Genève. Méprisé, laissé « en extreme necessité de 
ma noriture avec celle de toute ma famille l'expace de sis mois », il est dégagé à sa demande de ses obligations " pour ne souffrir plus tant du Sire Olivier de Serres, [...] que de plusieurs aultres ${ }^{3}{ }$. La fin de la lettre de Besson est un réquisitoire contre les protestants de Villeneuve qui, " après avoir tant importuné par le moyen du dict Olivier, tant vous que les aultres peres de Geneve, pour mavoir leur Ministre », l'ont fait venir pour le traiter «si durement apres ». " Dautant, rappelle-t-il, que javois assés predict a tous mon imbecillité avant que destre envoyé ${ }^{4} »$.

Si Jacques Besson ne semble pas avoir brillé comme pasteur, peut-être son intérêt pour l'étude des eaux souterraines s'est-il déclaré au cours de ces deux années de séjour à Villeneuve-de-Berg.

Dans L'art et science de trouver les eaux, publié en 1569, il énumère les signes trompeurs sur lesquels s'appuient habituellement agriculteurs et architectes pour trouver l'eau ${ }^{5}$. On trouve la même énumération de ces "signes frauduleux » dans le VII ${ }^{\mathrm{e}}$ Lieu du Theatre d'agriculture de Serres qui y dénonce «telles farfanteries, inventées pour confondre l'œuvre et se rendre admirables » et recommande plutôt se fonder « és choses asseurées, \& sur lesquelles, comme presque les touchant au doigt, l'homme d'esprit asserra solide jugement ${ }^{6} »$. Ces préconisations qu'il développe dans son chapitre $\mathrm{III}^{7}$ ressemblent fort à celles que Besson présente dans son Livre II.

Ceci posé, le propos de Serres se distingue immédiatement, signalant que, selon lui, il n'est pas question " de discourir de l'origine des fontaines, de leurs essors, de la propriété de leurs eaux ", « telle curieuse philosophie surpassant l'entendement de l'homme des champs, qui a plustost besoin d'eau pour sa maison que de paroles pour repaistre son entendement $^{8}$ ». Or c'est précisément le projet de Besson. Il s'agit bien pour lui de " discourir de l'origine des fontaines ", non sans succès puisqu'il est le premier à avoir présenté dans L'art et science de trouver les eaux... une version correcte

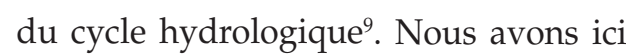
un aperçu de ce qui distingue la position de Serres, observateur curieux ${ }^{10}$, attentif aux expériences sensibles $q u$ 'il réalise pour asseoir son jugement et améliorer sa pratique "sans entrer en plus grandes spéculations ${ }^{11} »$.

L'influence de Besson, apothicaire, sur le jardin bouquetier et surtout le jardin médicinal ${ }^{12}$ est possible, comme celle de l'ingénieur sur les projets hydrauliques qui furent commencés au Pradel en 1564, peu après son départ de Villeneuve-deBerg. Il ne fut plus jamais question pour lui d'être pasteur.

Dans ce témoignage, Serres n'apparaît pas sous un jour très flatteur. Pour se faire une idée des valeurs qu'il défend, on peut aussi s'appuyer sur les traits qui se dégagent $\mathrm{du}$ personnage idéal qu'il a conçu, celui du "bon mesnager », qui dirige son domaine en père de famille ${ }^{13}$. Ce sont donc les rapports préconisés entre ce " père de famille » et son personnel que nous allons retenir.

Il est essentiellement un maître et tous ceux qui vivent ou travaillent sur le domaine sont définis par leur place dans la hiérarchie des subordinations, à commencer bien sûr par la mère de famille. Tous, fermiers, serviteurs, ouvriers, mercenaires, sont assujettis au " principe de divine police, de commander et d'obéir ». Pour éviter toute confusion, 
et que chacun connaisse sa place dans ces hiérarchies, elles sont rendues manifestes par des différences marquées et significatives " aussi bien au traitement, qu'aux habits et exercices », jusqu'aux nourritures qui sont distribuées selon le rang que l'on occupe dans cette hiérarchie. Une souveraine méfiance règne dans l'esprit du bon père de famille visà-vis de tous ses subordonnés, au point que les dispositions des parties de la maison inclinent à penser au Panopticon de Jérémie Bentham : l'œil et l'oreille du maître - et de la maîtresse - sont à l'affût et tout est fait pour une surveillance incessante, les serviteurs étant par nature " paresseux et déloyaux ». Cela va jusqu'à " tenir en vue comme en parade [...] tous ustenciles de cuisine fait de métal, pour que la mère-de-famille puisse vérifier leur bon entretien en un clin d'œil ». Quant au salaire, " tâcher de le rendre le plus petit possible ».

Le Pradel, domaine idéal, apparaît ainsi au fur et à mesure de la lecture comme un système très fortement hiérarchisé et même clos, afin d'éviter les rencontres fâcheuses des serviteurs avec le monde extérieur et ses turpitudes. De toute façon, comme l'affirme Olivier de Serres, " de tels gens ne sont guières capables de raison ${ }^{14}$ ", il convient donc de les traiter avec sévérité, mais sans la brutalité que Serres reproche à Caton, ceci au nom de la religion chrétienne ${ }^{15}$ et du "devoir de charité, d'honnesteté, de société ${ }^{16}$ ». Nonobstant, le «mauvais naturel de ses gens ${ }^{17}$ », " le sauvage et pervers naturel des mercenaires (ou de la plupart) » contraint le maître à la rigueur, alors même qu'il préfèrerait être débonnaire.
Faut-il voir, dans ce tableau de la méfiance, voire du mépris envers les subordonnés, une description conforme aux mœurs de l'époque? Peut-être est-il intéressant de comparer cette figure d'un administrateur sévère avec le personnage que campe Agostino Gallo dans ses Dialogues sur l'agriculture, lorsqu'il présente celui qui doit gérer son domaine. Gallo était en effet un propriétaire foncier et il présente son ouvrage comme le fruit de sa seule expérience dans la gestion de son propre domaine près de Brescia - il a alors 60 ans. Or son approche est beaucoup plus aimable et bienveillante que celle de Serres. En gros, il considère qu'on obtient le meilleur en étant soi-même bon et attentif aux autres, fût-ce des serviteurs, et il compte sur une fidélité encouragée par cette bienveillance. « Le bon père de famille \& sage mesnager est tousjours aymable, courtois, gratieux \& liberal envers ceux qui luy font service ${ }^{18}$. » On peut se demander si Serres ne lui répond pas lorsqu'il écrit : « Le doux traitement, le bien payer, le non-courroucer, le bon visage aux serviteurs, sont choses humaines et fort aisées à l'homme débonnaire; mais indifféremment employés, beaucoup préjudiciables à son service, pour le mauvais naturel de ses gens ${ }^{19}$. »

Le livre d'Agostino Gallo parut à Brescia en 1550 et connut très vite plusieurs éditions régulièrement augmentées en italien ${ }^{20}$ (vingt éditions), passant de dix à vingt livres, et fut traduit en français ${ }^{21}$ en 1571 . On peut s'étonner que les historiens qui s'intéressent à Olivier de Serres ne parlent pas de ce texte important qui présente bien des points communs dans ses analyses et ses exigences épistémologiques avec le Théâtre d'agriculture. 


\section{Le « Théâtre " d'agriculture}

Gallo écrit en forme de dialogue, ce qui était assez prisé à l'époque, même pour des ouvrages scientifiques (on peut penser à Galilée). Serres choisit la forme du «Théâtre " d'agriculture. Non seulement il intitule ainsi son ouvrage, mais encore il le divise non pas en "livres », comme c'est la coutume, mais en « lieux », ce qui est une manière d'insister : un " théâtre » divisé en " lieux », voilà qui renvoie précisément aux théâtres de mémoire. Ce qui est confirmé dans la préface :

"Mon intention est de monstrer, si je peux brièvement et clairement, tout ce qu'on doit cognoistre et faire, pour bien cultiver la terre et ce pour commodément vivre avec sa famille, selon le naturel des lieux, ausquels l'on s'habitue. Non que pourtant je veuille ramasser tout ce qu'on pourroit dire sur ce sujet : mais seulement disposer ès Lieux de ce Théâtre, les memoires de mesnage, que j'ai cogneu jusques ici estre propres pour l'usage d'un chacun, autant que ceste belle science y peut pourveoir ${ }^{22}$.»

Ainsi, l'allusion au théâtre de mémoire est très claire, même si le modèle a fait son chemin depuis l'Idea del teatro de Giulio Camillo23. À l'époque où Serres écrit, la notion de théâtre s'applique à toutes sortes de livres qui présentent des informations détaillées sur un sujet particulier selon un mode systématique et soigneusement ordonné, de manière à donner au lecteur à la fois une vue d'ensemble organisée et des moyens de se diriger dans le détail des connaissances. Cette métaphore du théâtre existe dans toutes sortes de domaines : appliquée à la vie humaine, c'est le théâtre moral; à l'ordre divin, c'est le théâtre de la nature, " témoignage éclatant de l'art de Dieu $^{24}$ ", écrit Melanchton. Les théâtres qui « représentent » des connaissances, comme l'écrit Serres, s'inscrivent dans un dispositif général de formalisation des savoirs pratiques dans et par l'écrit. À cet égard, le succès de la métaphore du « théâtre » est un des modes de réalisation d'une ambition qui s'exprime plus généralement à la Renaissance dans le projet de mise en ordre de pratiques selon un art, ou une méthode. En fait, tous les thèmes qui constituent ce qu'au $\mathrm{xVI}^{\mathrm{e}}$ siècle, on appelle "réduire en art ", les intentions qui portent cette " réduction », les effets que l'on en espère se trouvent dans l'ouvrage de Serres et $\mathrm{d}^{\prime}$ abord sont exprimés dans sa préface ${ }^{25}$. 


\section{Rassembler: le recours aux textes anciens et modernes}

Premier impératif et premier acte pour l'auteur du Théâtre d'agriculture : rassembler ce qui était éparpillé dans différents types de médias, écrits, images, proverbes, symboles, mais aussi gestuelle et transmission orale des pratiques de métiers, des connaissances ou des bribes de savoirs, appartenant à différentes époques et différents pays. Ce premier travail de rassemblement de données diverses ne saurait cependant se faire sans discrimination, sans choix raisonné. En outre, il faut immédiatement préciser que, l'ambition de Serres étant de produire un savoir utile, il n'est pas question de s'égarer dans les raffinements de connaissances spéculatives, mais de faciliter l'action et la production, de rendre opératoire toutes ces données qui se présentent selon une essentielle hétérogénéité et donc produire un ordre pour définir des domaines d'action, ce que Serres nomme « lieux », où dégager des catégories homogènes, condition de l'exercice d'une pratique contrôlable.

Des travaux récents se sont attachés à évaluer le recours aux textes anciens par Serres ${ }^{26}$ depuis Les travaux et les jours d'Hésiode, l'Économique de Xénophon, les Géoponiques de Cassianus Bassus, pour les Grecs; pour les Latins, les agronomes dont les éditions collectives se multiplient pendant le $\mathrm{XVI}^{\mathrm{e}}$ siècle : Caton l'Ancien, Varron, Columelle, Palladius, sans oublier les Géorgiques de Virgile et l'Histoire naturelle de Pline l'Ancien; parmi les auteurs modernes, L'Agriculture et maison rustique de Charles Estienne et Jean Liébault.
Ces écrits, affirme-t-il, demeurent indispensables :

«Il y en a qui se mocquent de tous les Livres d'Agriculture et nous renvoyent aux paysans sans lettres, lesquels ils disent estre les seuls juges competans de ceste matiere, comme fondée sur l'expérience, seule et seur reigle de cultiver les champs. [...] mais ceux qui nous renvoyent à eux seuls, me confesseront-ils pas, qu'entre le plus experimentés il y a divers jugements? et que leur expérience ne peut estre bonne sans raison ${ }^{27}$ ?»

Et de conclure que " qui ne void que l'experience des laboureurs non lettrés, est grandement aidée par la raison des doctes escrivains d'Agriculture ${ }^{28}$ ? »

Serres nous livre ici les sources et la méthode de son propre travail critique. En même temps, il rejoint une interrogation majeure, en débat à travers le temps parmi les auteurs de traités d'agriculture, à savoir s'il est possible de traiter en général d'un art qui s'exerce dans des conditions toujours particulières. La question, évoquée d'entrée par Columelle, est abordée par Gabriel Alonzo de Herrera ${ }^{29}$, auteur du premier grand traité d'agriculture de la Renaissance en langue vernaculaire, qui soutient que les différences locales ne sont pas raisons suffisantes pour rendre inopérants les savoirs transmis par l'écrit, et Serres reprend lui aussi le débat. 
Dans son Theatre d'agriculture, il ne recourra aux textes anciens traitant des différents lieux de l'agriculture que pour leur valeur à l'usage et, comme il le souligne fréquemment, parce qu'à l'usage répété ils se sont trouvés confortés. C'est l'une des figures de ce que l'on appelle « expérience ». Il faut rester attentif au fait que cette " expérience » est l'enregistrement par la mémoire de certains rapports entre une ou des actions et un ou des effets. Cela n'a rien à voir avec l'expérimentation scientifique, étant entendu que la finalité n'est pas la connaissance, mais l'utile, et qu'il n'y a pas lieu de parler de dispositif expérimental, avec ses contraintes, constantes et variables, comme on peut le voir déjà dans les traités de mécanique, par exemple. Toutefois, la répétition minutieuse d'expériences et la multiplication d'essais inédits transmis et commentés avaient déjà permis aux agronomes andalous, attentifs à respecter les équilibres entre les différents facteurs locaux, de constituer des domaines de savoir innovants ${ }^{30}$. Serres poursuit en ce sens avec le souci constant et revendiqué d'un discours tenu de bout en bout dans l'ordre du rationnel. Une réussite purement empirique n'est rien si elle n'est pas comprise, intégrée dans un savoir, à l'aide de la raison. Sans doute n'a-t-il pas expérimenté toutes les "expériences » qu'il nous livre, et il serait intéressant d'analyser ce qui lui permet de faire le tri dans l'énorme ensemble de données que lui apportent non seulement la littérature antique, mais aussi la littérature moderne dont il nous dit ne pas négliger la lecture, et les traditions artisanales ou paysannes parfois profuses dont il fait état.
Un premier filtre est celui qui écarte le merveilleux. Cependant celui-ci n'est pas forcément visible et distinct, de sorte que la méfiance déclarée de Serres visà-vis de ce qui excède l'expérience et la raison ne lui évite pas quelques dérives. Plus généralement, à la lecture de ce type d'ouvrage, on ne peut manquer d'être saisi par la facilité avec laquelle certains discours bien tenus et argumentés tout à coup s'élancent vers des témoignages, des preuves ou des expériences dites merveilleuses et nous projettent dans un espace intellectuel où nos frontières se brouillent. Voilà qui nous rappelle en quelque sorte à l'ordre. Ce monde intellectuel qui n'est pas le nôtre est parfois tout à fait familier et parfois difficile à saisir et apparemment incohérent.

Les traités anciens, surtout grecs et moyen-orientaux, sont constellés de merveilles qui cohabitent avec d'excellents raisonnements et la même revendication del'expérience. Àces procédés magiques, Olivier de Serres oppose la moquerie et il consacre trois pages de son traité ${ }^{31}$ à quelques préceptes « impertinents » tirés des Géoponiques, tant pour contenter la curiosité de "nostre mesnager " «que pour s'amuser à telles folies » et " afin que nostre père de famille quittant toutes ces vanités quoiqu'antiques, s'arreste à ce que par expérimentée raison et longue pratique verra estre bon à ses affaires et ouvrages $^{32} »$. 


\section{Écrire dans une langue accessible à tous}

Le deuxième point important à retenir pour définir le projet des agronomes de la Renaissance est celui de la langue. Écrire en langue vernaculaire pour atteindre le public concerné n'est pas seulement avoir à surmonter des problèmes de vocabulaire, mais, indissociablement, faire entrer les savoirs hérités des anciens dans la langue moderne est trouver le moyen d'exercer une critique. Dans son Obra de agricultura, Gabriel Alonzo Herrera s'adresse à son commanditaire ainsi :

« N'entendez aucunement que je dis être le premier inventeur de cet art d'Agriculture [...] je dis plutôt que je suis le premier qui ait essayé de mettre en castillan les règles et l'art de l'agriculture, dans lequel il est très pénible d'accorder les voix discordantes des auteurs, confronter, rejeter, choisir, réprouver quelques usages antiques ou modernes ${ }^{33}$. »

Cet effort critique sert un objectif : "L'art de bien employer et cultiver la terre en toutes ses parties, selon les diverses qualités et climats, tant par la doctrine des anciens et des modernes que par l'expérience. »

Herrera dit s'efforcer d'utiliser un langage commun, ce qui est confirmé par les historiens, et il le fait avec un grand souci de précision et d'ordonnancement dans l'exposés ${ }^{34}$ Son traité avait pour objet de favoriser la reprise de l'agriculture en Espagne après la Reconquista. Il connut au $\mathrm{XVI}^{\mathrm{e}}$ siècle douze éditions en langue espagnole et cinq traductions italiennes publiées à Venise. Celle de 1568 comprend des références à des auteurs modernes et, en particulier, à Agostino Gallo. Au temps où Serres écrit, il existe déjà un certain nombre d'ouvrages en français traitant d'agriculture, de botanique, d'élevage, et les auteurs anciens les plus courus ont été traduits en français. La question du vocabulaire technique ne se pose plus guère que pour la transcription des termes locaux.

Faciliter la lecture est aussi savoir retenir l'attention du lecteur. Les historiens ont souvent remarqué que, dans les traités d'agriculture qui se multiplient au $\mathrm{XVI}^{\mathrm{e}}$ siècle, la part de la rhétorique est aussi importante - parfois davantage que la part de connaissance effectivement en jeu. En effet, la rhétorique est un facteur essentiel de la transmission, qu'elle vise l'effet poétique sur le modèle des Géorgiques ou qu'elle s'efforce de souligner la scientificité du discours. Quant à la versification, ce peut être aussi un moyen de faciliter la mémorisation $^{35}$. Enfin, parmi les manières d'aider le lecteur et de le persuader, la méthode d'exposition mise en œuvre dans le livre est essentielle. 


\section{Ordonner méthodiquement}

Dans les théâtres de mémoire et singulièrement dans celui de Camillo, les idées, les notions, les concepts retenus tiennent leur pouvoir de connaissance de la place relative des lieux qu'ils occupent, par le système de relations possibles ou induits dans le dispositif scénique des savoirs. Dans l'économie du livre, c'est-à-dire la distribution de ses parties, la transmission du savoir s'exerce grâce à la formalisation du discours selon une méthode. Cette formalisation du discours a des traits affirmés dans tous ces écrits à visée pratique qui se répandent au cours du $\mathrm{XVI}^{\mathrm{e}}$ siècle : idéalement, il convient de donner au lecteur les moyens de vérifier la validité des raisonnements et des expériences en recourant, quand c'est possible, aux mathématiques et, plus généralement, à la dialectique qui se traduit en particulier dans ces ouvrages par l'usage de la dichotomie. C'est la méthode d'exposition de Serres : chaque lieu - c'est-à-dire chacun des huit livres qui composent le traité - est précédé d'un tableau dichotomique qui organise l'ensemble de ce qui va être traité dans cette partie selon un emboîtement hiérarchisé qui, de gauche à droite, procède du général au particulier, mais aussi, de haut en bas, sous la division majeure entre naturel et artificiel. Cette méthode d'investigation est caractéristique de la conception pratique, de la pensée pour l'action. La dichotomie est généralement vantée alors pour sa valeur tant mémorielle que cognitive; elle est une aide à la décision, à l'invention, comme on peut le voir dans la méthode de Simon Stevin ${ }^{36}$ ou les recommandations pédagogiques de Pierre Ramus ${ }^{37}$. La présentation selon des tableaux dichotomiques en tête des Lieux du Thêâtre d'agriculture se poursuit dans le texte par la mise en œuvre de couples d'opposés, diligence/négligence, profit/plaisir, nécessité/contentement, etc. qui structurent le discours de Serres et guident le jugement du lecteur dans une certaine direction. Conduire l'esprit, ouvrir une voie dans la connaissance, c'est la définition même de la méthode. Faire travailler ces " contrariétés " est l'un des moyens dont nous disposons pour saisir ce qui nous est utile, pour opérer des choix dans la complexité d'une nature diverse et souvent insaisissable de par la volonté divine elle-même.

Le principal de ces couples concerne l'origine des connaissances que le livre transmet, soit les livres et la pratique, et dans les livres les anciens et les modernes, dans la pratique l'expérience directe et l'expérience indirecte (ce qu'il a vu faire sans le faire). Or les contenus des connaissances qui viennent de ces différentes sources ne se recoupent que partiellement. Il est certain qu'une très grande partie de l'exposé du Theatre d'agriculture - et des théâtres de savoirs de l'époque - est tirée de lectures, lectures croisées, associées ou opposées, confrontées à leurs propres singularités : en agriculture, ce que dit l'un ou l'autre selon le pays ou le climat ou la qualité des terres où il se trouve et dont il fait état. Le champ des lectures est aussi un champ de réflexion et de critique : làdessus, tous ces traités techniques sont formels - nous avons vu que c'est le cas de celui d'Herrera - le savoir hérité des anciens déjà en lui-même contient les 
germes de la critique par la seule variété des sources.

Enfin, plus essentiellement, tous ces écrits se trouvent confrontés à une question majeure et alors vivace dans tous les domaines des productions humaines, qui est celle du rapport entre art et nature. L'art est-il confiné à l'imitation de la nature, ou peut-il dépasser la nature? On trouve chez les agronomes des paroles étonnantes qui font la part belle à l'art humain. C'est en particulier le cas de la greffe : il faut rappeler que l'émerveillement devant l'admirable artifice de la greffe, comme dit Serres, est aussi ancien que les écrits sur l'agriculture. Herrera ose écrire : " Enter est chose admirable, il semble que de cette façon nous rivalisons pour égaler la nature, et encore nous la dépassons plus d'une fois, ce qu'elle rend mauvais s'améliore avec la greffe et le bon devient meilleur ${ }^{38}$. »

Ce type d'expérience conduit l'homme de l'art à la vigilance; il doit se tenir prêt à de nouvelles expériences, scruter les événements pour y découvrir des moyens d'augmenter les profits du

\section{Sensible et intelligible}

Dans le contexte de l'art ou science pratique qui se construit alors, il semble important de garder à l'esprit que le rapport entre sensible et intelligible, tel qu'il se présente dans ces écrits, est à la fois au centre de la réflexion et demeure très instable à l'intérieur d'une même œuvre. Nous avons vu Serres très attaché à la sanction du sensible et assez méfiant, dès que le raisonnement cède devant la spéculation. Cette attitude qu'il main- domaine. Selon Serres, l'une des qualités qui font le «bon mesnager » est la capacité à se saisir d'une découverte fortuite : " le bon mesnager emploiera l'occasion ». «Plusieurs belles et rares choses sont venues en lumière par accident qui, rédigées en art, ont donné de grands profits », souligne-t-il. C'est vrai de la couvaison des poules, de l'invention des champignons de couche ${ }^{39}$. Un autre exemple concerne une invention dont il est très fier, " touchant la cognoissance de la faculté de l'escorce de meurier ». On s'en sert habituellement pour faire des cordes et puis, par accident, l'écorce mise à sécher tombant dans l'eau, ramassée, lavée, est apparue la matière molle et douce propre à filer : " voilà la première espreuve de la valeur de l'escorce du meurier blanc, lequel accident rédigé en art, n'est à douter de tirer bon service de telle invention ${ }^{40} »$.

Notons que cette importance donnée à l'occasion est caractéristique des réflexions menées à cette époque sur l'action, ses modalités, sur l'invention et la méthode d'inventer.

tient avec constance n'est pas sans lui jouer des tours. Pour en donner une illustration, je propose que l'on s'arrête sur la proposition qu'il fait pour trouver la surface d'un rond. Il écrit : « Encore bien que le rond aye beaucoup travaillé jusqu'ici tous les géométriens, antiques et modernes, si est-ce qu'en confessant y avoir employé quelques heures, j'en dirai en passant mon avis, comme un de la foule ${ }^{41}$. » Cet avis est « une nou- 
velle procédure que je tiens pour y parvenir, promettant d'en recevoir une autre, quand elle me sera monstrée estre plus asseurée ", procédure que Serres confesse être " une opération et preuve mécanique et incognue à la géométrie, n'en recevant point ses démonstrations; mais aussi est-elle autant éloignée de fausseté, comme facilement, par le sens naturel elle peut estre comprise. »

Ce privilège accordé aux perceptions sensibles sur le raisonnement démonstratif a de quoi surprendre à l'époque où il écrit et par rapport à ses positions de principe. La question est : comment réduire un rond en une figure carrée de même superficie. La solution : prendre le côté du triangle équilatéral inscrit dans ce rond, le multiplier par lui-même, ce qui équivaut à la surface d'un carré qui, selon Olivier de Serres, est à très peu près celle du cercle. La preuve "mécanique » est la suivante : selon la méthode précitée, tracer le rond et le carré correspondant dans une matière bien unie comme du papier ou du parchemin et les placer sur les deux plateaux $\mathrm{d}^{\prime}$ une balance : ils ont à très peu près le même poids, donc la même surface ${ }^{42}$.

Ce type de procédé est intéressant si l'on se souvient qu'il appartient à une tradition médiévale ${ }^{43}$, celle de la géométrie pratique, étudiée par Victor Mortet et présentée par Paul Tannery, historien des mathématiques selon lequel

« il est clair que si les lettrés du $\mathrm{xI}^{\mathrm{e}}$ siècle connaissent traditionnellement, par exemple, la formule d'Archimède pour le calcul de l'aire du cercle, ils ne peuvent pas imaginer qu'elle ait été obtenue autrement que par des procédés empiriques, "in membranis et pelliculis", dans des parchemins et des peaux. Il est alors commun de parler de vérification graphique effectuée en découpant des morceaux de parchemin ».

C'est bien le cas ici. Toutefois dans le même chapitre, Serres fournit tous les éléments de géométrie et d'arithmétique pratique qui permettent de «ne se laisser decevoir aux commis ès impositions ${ }^{44} »$. Pour nous autres lecteurs, toujours tentés de tirer vers l'avant les procédés alors en œuvre, la coexistence de ces deux types de preuve ou de vérification a l'avantage de nous ramener à plus de précautions dans l'appréciation de la rationalité en œuvre.

\section{Olivier de Serres et la sériciculture de son temps}

L'élevage des vers à soie occupe une place particulière dans l'œuvre d'Olivier de Serres. La cueillette de la soye par la nourriture des vers qui la font. Échantillon $d u$ Theatre d'Agriculture d'Olivier de Serres, seigneur du Pradel, sortit de presse " le dix huitième jour de février M.D.XCIX [1599] » et devait constituer le cha- pitre $\mathrm{XV}$ du cinquième Lieu du Theatre d'agriculture ou mesnage des champs. Serres avait hâté la publication de cette partie de son traité à la demande du roi. Laffemas ayant rédigé en 1598 son édit sur le commerce d'inspiration mercantiliste, diminuer les importations de soie et en produire dans le royaume en 
quantité allaient dans le sens de réformes envisagées. La culture du mûrier blanc dans le nord de la France devint une affaire d'État et Serres fut chargé de pourvoir à ces plantations en particulier en Île-de-France ${ }^{45}$. On sait qu'il a voyagé en Europe et visité des magnaneries dans le Languedoc, mais il existait aussi toute une littérature sur cet élevage sur laquelle il faut s'arrêter, ne serait-ce que pour apprécier ce qui distingue ou simplement caractérise son approche.

Notons d'abord que cette littérature est tardive. En effet, l'élevage du ver à soie, introduit à Byzance en 552-554, a gagné l'ensemble du pourtour méditerranéen très progressivement et selon le même procédé : par l'enlèvement de femmes ou de jeunes gens avertis des pratiques de cette délicate " éducation " pour l'introduire et la développer; il n'y avait pas d'autre moyen. En effet, les soins à apporter pour cet élevage n'avaient pas donné lieu à une transmission écrite en Occident et pas (ou peu) davantage dans le monde arabe ${ }^{46}$. Si le calendrier de Cordoue, au $x^{\mathrm{e}}$ siècle, signale les mois importants pour commencer la couvaison des œufs et les soins à apporter selon les périodes de métamorphose des vers ${ }^{47}$, ce sont des ouvriers venus de Syrie qui introduisirent la sériciculture en Andalousie. En 1147, durant la deuxième croisade, le roi normand Roger II de Sicile attaqua Corinthe et Thèbes, deux importants centres byzantins de production de la soie, et déporta des ouvriers à Palerme. L'industrie normande de la soie fut ainsi le point de départ de l'industrie italienne puis de la française, toujours selon le même vecteur de transmission des techniques d'élevage, par les praticiens eux-mêmes.
Plus tardivement, pour les introduire en Nouvelle Espagne, on fit venir des ouvriers et surtout des ouvrières d'Andalousie, région où l'art de la soie s'était considérablement développé au Moyen Âge. Il faut attendre la fin du $\mathrm{xv}^{\mathrm{e}}$ siècle et surtout le $\mathrm{xvI}^{\mathrm{e}}$ siècle pour que cette activité donne lieu à des écrits destinés à en divulguer les méthodes. Olivier de Serres, avec son opuscule de 1599 , se situe à la fin d'un siècle qui a vu se développer et se perfectionner toute une littérature sur le sujet - en gros une vingtaine d'écrits. Ils sont pour la plupart italiens, mais on traduit vers le français et aussi de l'espagnol et du français à l'italien. On peut donc considérer que ces connaissances se répandent et se répondent par le livre dans l'Occident de la Renaissance. Ainsi, lorsque Serres écrit son petit traité, il dispose d'une tradition écrite tout à fait élaborée.

Les premiers textes sont en vers latins et présentent, sur le mode virgilien du poème didactique ${ }^{48}$, avec les références mythologiques, astronomiques ou religieuses requises, des descriptions des différentes phases de l'élevage. La valeur informative de ces poèmes, non négligeable, n'est guère utilisable dans la pratique ${ }^{49}$. Le genre poétique se poursuit dans la seconde moitié du siècle et au début du $\mathrm{XvII}^{\mathrm{e}}$, avec La Sereide d'Alessandro Tesauro en $1585^{50}$, puis l'Histoire des vers qui filent la soye de Beroald de Verville en $1600^{51}$, mais, cette fois, c'est en langue vernaculaire, comme le sont les autres écrits, traités ou dialogues publiés qui se veulent d'un abord facile. C'est le cas en 1564 de l'Avertimenti [...] bellissimi, et la seta de Levantio da Guidicciolo $^{52}$. Un an plus tard, Agostino Gallo publie Le Dieci giornate della vera 
agricoltura ${ }^{53}$. Nous avons vu qu'au cours des ans, il complète son ouvrage et son édition de 1569 comprend déjà une seizième journée " nella quale si tratta de'Cavalieri, ò Bachi, ò Vermi ${ }^{54}$, che fanno la seta ${ }^{55}$ ».

Ces écrits se multiplient dans les années 1580-1590 et certaines prennent $\mathrm{du}$ volume. Ainsi, Corsuccio da Sascorbaro, passant en revue ses prédécesseurs, affirme qu'il n'y a pas grandchose à en tirer et, malgré leur célébrité, reproche aux vers latins de Monseigneur Vida d'être trop brefs et obscurs. S'il utilise Gallo parmi les auteurs qu'il cite dans son ouvrage, Il vermicello dalla seta ${ }^{56}$, c'est pour justifier aussitôt ses emprunts : "Solamente ho inimitato il Gallo da Brescia in alcuna cosa, e non credo che mi sia imputato à vitio, perche questo su, e sempre sarà costume de compositori, chi vedrà l'uno, e l'alto, potra dare chiara sentenza. »

La partie de son livre consacrée à l'élevage des vers à soie, qui s'adresse aux dames de Rimini, est très détaillée, appuyée, dit-il sur la " chiera esperianza ${ }^{57}$ ", et sans doute plus minutieuse dans son développement que l'ouvrage de Gallo, mais c'est quand même bien l'approche de ce dernier qui s'avère la plus moderne par son ancrage dans la pratique et l'observation directe des techniques pratiquées ${ }^{58}$.

Nouveauté et perfectionnements sont revendiqués dans ces écrits. C'est le cas des Dialoghi di M. Magino Gabrielli Hebreo venetiano sopra l'utili sue invenzioni circa la seta (Venise, 1588) qui proposent un moyen de faire deux couvaisons par an. On voit que le sujet a acquis un nouvel intérêt dans le public. Non seulement, il est divulgué en langue vernaculaire dans des textes souvent courts (quelques dizaines de pages), mais ils sont ajoutés à des textes plus généraux sur l'agriculture et l'élevage, comme on l'a vu chez Gallo, ou encore à des traductions, comme le fait Ercole Cato qui traduit Charles Estienne ${ }^{59}$ et ajoute des éléments nouveaux propres à l'Italie, en particulier sur le mûrier ${ }^{60}$.

Si l'Italie se distingue au $\mathrm{XVI}^{\mathrm{e}}$ siècle par le nombre et la qualité des écrits sur l'agriculture et en particulier sur notre sujet, la Obra de agricultura de Herrera est enrichie d'apports dus aux agronomes d'al-Andaluz. Comme eux ${ }^{61}$, Herrera ne fait allusion à l'élevage des vers à soie qu'à l'occasion de sa présentation du mûrier et il faut attendre l'édition posthume de 1620 pour qu'y soit adjoint le premier traité de sériciculture écrit en espagnol, Arte nuevo para criar seda, desde que se revive una semilla, hasta sacar otra, compuesto por Gonçalo de las Casas, paru à Grenade en $1581^{62}$. L'auteur y revendique pour sa famille l'introduction de cet élevage au Mexique, dans la Mixtequa Alta, et semble-t-il à juste titre ${ }^{63}$. Cet ouvrage se distingue par une approche tournée vers la connaissance du ver à soie et qui ne se borne pas simplement à ce qui est indispensable pour son élevage. Il affirme traiter de la théorie et des manières de la mettre en pratique, " la teorica y como se ha de poner en pratica ${ }^{64} »$. Ainsi, là où Gallo et Serres se contentent d'une description très élémentaire de l'animal, Las Casas procède à une véritable leçon d'anatomie, et d'anatomie comparée $\mathrm{d}^{\prime}$ ailleurs, pour mieux se faire entendre du lecteur. C'est un traité érudit, documenté - l'auteur indique ses sources ${ }^{65}$ et très intéressant en ce qu'il reste au plus près de pratiques marquées par un environnement humain, géographique, 
social, politique, particulier. Il sait très bien faire face à l'un des problèmes que pose tout traitement d'une pratique locale dans un ouvrage qui se veut aussi d'une portée générale. C'est, nous l'avons vu, la question constamment rencontrée depuis l'antiquité dans les écrits sur l'agriculture : comment être à la fois valable partout et utile ici et maintenant, dans des conditions toujours particulières. Ceci dit, ce traité demeure très scolastique dans sa forme et sa rhétorique et multiplie les références à l'action divine et aux vertus chrétiennes. Ainsi, la volonté de faire œuvre de divulgation des connaissances relève selon lui d'une mission impartie par Dieu à l'homme vis-à-vis de l'homme, œuvre de charitéé ${ }^{\prime}$. Pour le protestant Olivier de Serres, ce sera pour le bien public, le profit de l'éleveur et à la gloire de Dieu.

L'élevage du ver à soie a donné lieu à tant de commentaires et digressions que le sujet mériterait un traitement plus approfondi. Les métamorphoses de ces vermisseaux ont enflammé l'imagination de mystiques soufis et chrétiens, comme Thérèse $d^{\prime} A^{\prime}{ }^{2}{ }^{67}{ }^{67}$. Il est surtout associé aux femmes, ses meilleures " conductrices » et à la féminité ${ }^{68}$, celle plutôt des vierges, et surtout en se méfiant de celles qui ont "leurs fleurs" ou " règles de leurs temps " (Béroald de Verville). Les femmes jouent un rôle essentiel puisque, non seulement, elles sont les plus habiles et légères dans leurs gestes, mais c'est entre leurs seins que les graines sont couvées, là-dessus l'accord est unanime depuis Lazzarelli, Marco Vida, Tesauro jusqu'à Beroald de Verville qui affirme que " la chaleur que l'amour a mis en ces beaux seins " permet une éclosion « hastive ». Cette solution, très commune en Espagne selon Las Casas, est préconisée ou au moins envisagée aussi par tous les autres auteurs ${ }^{69}$, à l'exception de Serres pour lequel « couver ceste graine sous les aisselles, ou entre les mamelles des femmes, n'est chose profitable; non tant pour crainte de leurs fleurs, comme aucuns pensent, mais pour l'agitation : ne pouvant se faire que portant la graine sur sa personne, l'on ne la tracasse et meslinge $\mathrm{e}^{70}$ ».

Cet élevage étant ignoré dans les écrits des Anciens, il a fallu que le $\mathrm{xvI}^{\mathrm{e}}$ siècle invente une mythologie et c'est Vénus qui est requise tant pour l'apparition du mûrier que pour l'élevage des vers. La tradition aussi propage quelques pratiques évidemment fausses mais pérennes. Il en est une particulièrement présente et par rapport à laquelle les différents auteurs se déterminent, c'est celle de l'engendrement de vers par le pourrissement d'un veau enfermé dans une étable, uniquement nourri de feuilles de mûrier, tué et livré à la décomposition. Des vers apparaissent sur son cadavre et, dit-on, ce sont des vers à soie. Vida y souscrit ${ }^{71}$, Corsuccio s'élève contre ce prétendu engendrement du ver à soie ( nato di corruttione, o putredine $)^{72}$, Gallo « qui n'escrit rien, que luy mesme n'aye pratiqué ${ }^{73}$ ", n'y fait même pas allusion; quant à Las Casas, il s'insurge : comme tous les animaux, le ver naît de l'accouplement du mâle et de la femelle, " y no de corrupcion de ternera, o vaca, como algunos quieren dezir ${ }^{74} »$. À la fin de son ouvrage, il revient sur le sujet des croyances de ce type, les dénonce encore et ajoute : " a mi me parece que dezir esto, es un genero de alquimia, y muy semejante a $\mathrm{el}^{75} »$. 
Serres consacre de nombreuses pages dans plusieurs Lieux de son Théâtre d'agriculture à dénoncer les pratiques à caractère magique et pourtant, curieusement, il maintient dans son texte, à propos de l'obtention de la "graine " de ver à soie, cet étrange moyen $\mathrm{d}^{\prime} \mathrm{y}$ pourvoir :

«Ici est à propos de montrer le subtil artifice que l'homme a inventé pour réparer le défaut de graines et semences des vers à soie, avenant qu'elle soit perdue. Chose tirée des secrets de la nature et recherché avec grande curiosité [...]. Au printemps un jeune veau est enfermé dans une étable petite, obscure, sèche, et là nourri avec la seule feuille de murier, vingt jours durant sans nullement boire, ni manger autre chose durant ce temps-là au bout duquel est tué et mis dans une cuve pour y pourrir. De la corruption du corps sort abondance de vers à soye $\mathrm{e}^{76}$. »

La façon dont Serres aborde le sujet - " ces choses ", écrit-il, prenant ainsi ses distances - sont intéressantes précisément parce que, allant à l'encontre de la méthode fondée sur l'expérience dont il se réclame, il va malgré tout non seulement en rendre compte, mais en rendre raison. Donc, première précaution, sa connaissance n'est que d'ouie dire : « je vous représente ces choses sous le crédit $\mathrm{d}$ 'autrui en attendant que la preuve me donne matière de vous asseurer de ce qui en est ». Il se plaint alors que ses prédécesseurs n'en aient pas fait l'expérience et ajoute : "Je vous représente, dis-je, ces choses, à ce que rencontrant vraie telle création de vers-à-soye, et y treu- vant de l'avantage, soyons délivrés de la peine d'en envoyer cercher la semence en Espaigne et ailleurs, renouvellant le souci de s'en pourveoir par chacune année $^{77}$. " Première raison donc, toute pratique, de prêter l'oreille à ces choses, la difficulté de se procurer annuellement de nouvelles semences. La suite du texte qui vise à la justification, déploie toutes sortes de circonlocutions : Olivier de Serres ne l'abordera que "s'il est question de discourir là-dessus ", seraient-ce ses lectures qui l'y poussent? Et alors, si l'on n'a pas de preuve de véracité, « je dirai, tel engendrement de vers-à-soye n'estre mescroyable (notons la forme précautionneusement négative) puisque toute corruption est commencement de génération ». En effet, poursuit-il, comme on peut l'observer tous les jours, " des choses corrompues sortent diverses vermines ", ce que l'on ne peut nier, mais Serres ajoute: " selon les diverses qualités des matières ». Cette dernière incise prend appui sur l'Écriture (du taureau et du lion s'engendre l'abeille) et les Anciens qui " tiennent que du cheval et du mulet s'engendrent les guespes », etc., " partout nous voyons la Nature créer des bestioles, vermisseaux, moucherons avec autant d'admiration qu'admirable est le créateur ».

Ceci posé, peut-être bien que douter de la création des vers à soie par le procédé en question serait un manque d'admiration pour les créations de Dieu, in fine un manque d'admiration pour le créateur. Ce dernier argument devrait justifier que Serres s'y arrête en attendant preuve. Reste que les raisons qu'il invoque, appuyées sur une vague scolastique aristotélicienne et le recours à l'Écriture, ne peuvent manquer de nous 
faire penser qu'une méfiance proclamée pour la réflexion théorique peut favoriser d'étonnantes errances, mais aussi en éviter d'autres : à la fin de son chapitre sur les vers à soie, il explique que, tenté d'entrer dans des secrets de nature, il s'en gardera, précisant : « ainsi mes discours ne s'enfonceans jusques au centre, $s^{\prime}$ arresteront à la superficie ${ }^{78} »$. Mais, plus que ces évitements, tentations ou dérives contrôlées, ce qui frappe à la lecture de ce qui est un vrai manuel pour un éleveur et producteur de soie, est l'ensemble des précautions que l'on pourrait dire hygiénistes qu'il préconise, plus que les autres auteurs de tels ouvrages sans doute et avec un grand luxe de précisions. L'autre aspect remarquable de sa présentation est son attention à la gestion technique et financière de ce type d'élevage. Ce qui est vrai de toute son œuvre.

Dans sa préface, Serres exposant les raisons qui l'ont poussé à écrire et « gayment représenter ceste belle science d'agriculture ", dit l'avoir voulu faire " pour contribuer [...] au vivre des hommes ».
«Consacrer leur vie à augmenter les revenus des biens reçus de leurs ancêtres, du même coup, acquérir ainsi l'honneur d'avoir vertueusement vécu en ce monde une belle vie - richesse à priser par sur toute autre - c'est ce à quoi parviendront les bons ménagers, par la bénédiction de Dieu. »

C'est par ces mots qu'il clôture son œuvre, avec des termes qui auraient sans doute enchanté Max Weber ${ }^{79}$.

Mais encore, par rapport aux textes anciens auxquels il se réfère, sa modernité est particulièrement à l'œuvre dans son attention à ce qui conduit l'esprit humain vers la résolution contrôlée des problèmes pratiques de l'agriculture. Ce n'est pas seulement un traité de l'agriculture, c'est un traité des capacités, infirmités et pouvoirs de l'esprit humain face à la confrontation d'un héritage savant considérable et de l'expérience des choses. 


\section{Annexe}

\section{Gabriel Alonso de Herrera, Libro de agricultura..., Alcala de Henares, 1539, Prologue n. p.}

La première règle de cet ouvrage est que pour bien faire, dans cet ouvrage comme dans tous les autres, trois choses sont requises : le savoir, le pouvoir et le vouloir et si l'une vient à manquer rien ne va. Parce que tout ouvrage où ces trois choses concourent sera parfait : et si l'on voit juste sans l'une d'elles, ce sera par hasard.

La deuxième règle dépend $d^{\prime} u n e$ partie de la première qui est du savoir : je dis que celui qui s'adonne aux travaux des champs, et nonobstant à quelque autre métier doit avoir l'expérience, et si à l'expérience il peut joindre la science et l'art, c'est beaucoup mieux : mais si l'un vient à manquer, il vaut mieux l'expérience sans l'art que l'art sans l'expérience, parce que l'expérience bien appliquée se trompe rarement, c'est pourquoi on affirme qu'il faut faire confiance à l'art de l'artisan, surtout s'il est bien expert.

La troisième règle est parce qu'en tout métier, et principalement en celui-ci, réussit bien celui qui s'y consacre. Il est très nécessaire qu'une telle personne ait du goût pour son métier parce que l'inclinaison fait le bon artisan. Et ainsi les métiers des champs veulent que le cultivateur ait de l'inclinaison pour eux, les apprécient et les honorent. Car il est bon que chacun apprécie le métier qui l'entretient et il y réussit très bien en y mettant toute sa diligence. Surtout dans les choses de la campagne qui n'admettent ni bons à rien ni paresseux.

Et je ne veux pas manquer d'énoncer une autre règle essentielle avec les précédentes : que tout homme doit éviter le travail des mauvaises terres s'il n'y est pas contraint car que le coût sera plus grand et le profit plus petit. Et il pourra l'appliquer à quelque usage qui sera moins coûteux et plus profitable. 


\section{Notes}

1. Olivier de SERRES, Le theatre d'agriculture et mesnage des champs, Paris, Jamet-Métayer, 1600; on compte 25 éditions, édition citée ici : Arles, Actes Sud, 2001.

2. Luisa Dolza et Hélène Vérin, " Dal livre al theatrum di Jacques Besson ", introduction à $\mathrm{Il}$ Theatrum instrumentorum et machinarum di Jacques Besson, Roma, ed. dell'Elefante, 2001, p. 3-49.

3. Eugénie Droz, "Jacques Besson, ministre de la parole de Dieu et ingénieur », Chemins de l'hérésie. Textes et documents, t. IV, Genève, Slatkine, 1976, p. 287-288.

4. Ibid.

5. Dans L'art et science de trouver les eaux... (préface, n. p.) ; pour plus de détails, voir Besson, notice sur le site Architectura du CESR [http://architectura.cesr.univ-tours.fr/traite/Auteur/Besson. asp ?param=>].

6 . O. de SERres, Le theatre, op. cit., p. 1119.

7 . Ibid.

8 . Ibid.

9. Découverte que Bernard Palissy s'attribue dans son Discours admirable de la nature des eaux et fontaines..., Paris, Martin le Jeune, 1580 ; Jean MARGAT, Didier Pennequin et Jean-Claude Roux, Histoire de l'hydrogéologie française, Association internationale des hydrogéologues, Comité français de l'hydrogéologie, 2013 (en ligne, <www.amicalebrgm.fr/ v3b/IMG/pdf/presentation_histoire_hydrogeo$\operatorname{logie}>$ ).

10. Serres emploie ce terme dans le sens de « celui qui prend soin de $»$.

11. O. de SERrEs, Le theatre, op. cit., p. 86.

12. Ibid., ch. XII et XIV du sixième Lieu; Jacques BESSON, Art et moyen parfaict de tirer huyles et eaux de tous médicaments..., Paris, Galiot du pré, 1573.

13. O. de SErres, Le theatre, op. cit., Lieu I, chapitre VI.

14. Ibid., p. 123.

15. Caton l'ancien, De re rustica, vers - 160 ; il s'agit du traitement des esclaves sur le domaine.

16. O. de SERres, Le theatre, op. cit., p. 121.

17. Ibid., p. 122.

18. Secrets de la vraye agriculture, et honnestes plaisirs qu'on reçoit en la mesnagerie des champs, pratiquez et experimentez tant par l'autheur qu'autres experts en ladicte science, divisez en XX journées, par Dialogues, Paris, Chesneau, 1571, p. 11.

19. O. de SERres, Le theatre, op. cit., p. 122.

20. Le Dieci giornate della vera agricoltura e piaceri della villa di M. Agostin Gallo, in dialogo, et Le Vinti giornate dell' agricoltura et de piaceri della villa...

21. Le traducteur est François de Belleforest.
22. O. de Serres, Le theatre, op. cit., Préface, p. 61.

23. Publié en 1550; Frances A. Yates, L'art de la mémoire, Paris, Gallimard, 1975; Paolo Rossi, Clavis universalis, Grenoble, Éd. Jérôme Millon, 1993.

24. Philippus Melanchton, Initia doctrinae physicae, Wittenberg, Johannes Lufft, 1549, p. 1.

25. Pascal Dubourg Glatigny et Hélène Vérin, Réduire en art. La technologie de la Renaissance aux Lumières, Paris, MSH, 2008.

26. Martine GorrichoN « Sources latines d'Olivier de Serres ", Bulletin de l'Association d'étude sur l'humanisme, la réforme et la renaissance, $\mathrm{n}^{\circ} 50$, 2000 , p. $45-58$.

27. O. de SERres, Le theatre, op. cit., p. 63.

28. Ibid., p. 64.

29. Gabriel Alonso de Herrera (1470-1539), Obra de agricultura copilada de diuersos auctores por Gabriel Alonso de Herrera de mandado del muy illustre y reuerendissimo Señor el cardenal de españa arcobispo [sic] de Toledo, Alcala de Henares, Arnao Guillen de Brocar, 1513.

30. C'est le cas de l'extraordinaire sophistication des règles de production et d'utilisation des engrais; voir Lucie Bolens, «De l'idéologie aristotélicienne à l'empirisme médiéval. Les sols dans l'agronomie hispano-arabe ", Annales ESC, 1975, vol. 30, p. 106108; Jean Boulaine, Histoire des pédologue et de la science des sols, Paris, INRA, 1988. Il faut ici citer IBN Bassal et IBN Al'Awwan, Le livre de l'aAgriculture, Arles, Actes Sud, 2000.

31. O. de Serres, Le theatre, op. cit., p. 132-134.

32. Ibid., p. 134.

33. « No entienda ninguno que digo ser yo el primer inventor de esta arte de Agricultura... mas digo ser yo el primero que en castellano procuré poner las reglas y arte dello, lo cual cuánto sea trabajoso concordar a las veces discordes autores, cotejar, desechar, escoger, reprobar algunos usos antiguos y modernos, vuestra ilustre señoría lo ve... ", G. A. de Herrera, Obra de agricultura..., op. cit., p. 6.

34. Consolación BARAnda, "Cienza y humanismo : la Obra de agricultura de Gabriel Alonzo de Herrera (1513) », CRITICÓN, 46, 1989, p. 95-108.

35. C'est vrai aussi pour Ibn Luyun, agronome andalou (1282-1349), qui écrit, dit-il, son Traité d'agriculture en vers rajaz, le plus simple et populaire, dans le but de répandre le plus possible les connaissances agricoles, Ibn Luyun : Tratado de agricultura, Granada, Publicaciones del Patronato de la Alhambra y el Generalife, 1988, traduction de l'arabe de J. E. IBÁÑEZ.

36. Les cuvres mathématiques de Simon Stevin..., Leyde, B. \& A. Elsevier, 1634. Le Livre de la géographie, p. 126, col. 2. 
37. Il faut rappeler aussi que c'est un procédé mis en œuvre systématiquement par les théologiens protestants.

38. "Es cosa maravillosa el enxerir, que parece que con ello contendemos en igualarnos con la natura, y aun la emendamos muchas veces, que lo que ella hace malo con el enxerir se emienda y lo bueno mejora... ", G.A. de Herrera, Obra de agricultura..., op. cit., p. 120.

39. «La nature incite les poules à couver et eslever leurs petits; aidant aux poules, leur inclinaison naturelle a esté redigée en art », O. de SERRES, Le theatre, op. cit., p. 569; à propos de champignons, ibid., p. 873.

40. Ibid., p. 771-772. On ne peut manquer de rapprocher cette invention de celle attribuée en Chine à l'impératrice Leizu : un cocon de ver à soie serait tombé dans sa tasse de thé, d'où elle aurait tiré un fil, aurait eu l'idée de le tisser, etc. L'édition du Théâtre de 1603 et les suivantes comprennent au Livre $\mathrm{V}$ un chapitre XVI, " la préparation de l'Escorce du Meurier blanc, pour en faire du linge et autres ouvrages ». Le contenu de ce chapitre fit l'objet d'un petit opuscule (28 p.), La seconde richesse du meurier blanc, qui se trouve en son escorce pour en faire des toiles de toutes sortes, non-moins utiles que la soie, provenant de la feuille d'iceluy. Eschantillon de la seconde édition du Théâtre d'Agriculture, d'Olivier de Serres..., Paris, Abraham Saugrain, 1603.

41. O. de SERres, Le theatre, op. cit., p. 85.

42. Ibid., p. 86.

43. Paul TANNERY, "Une correspondance d'écolâtres du $\mathrm{xI}^{\mathrm{e}}$ siècle ", dans Comptes rendus des séances de l'Académie des inscriptions et belles-lettres, $41^{\mathrm{e}}$ année, $\mathrm{n}^{\circ} 2,1897$, p. 214-221; il présente l'article de Victor Mortet, Note historique sur l'emploi de procédés matériels et d'instruments usités dans la géométrie pratique au Moyen Âge (X'-XIII siècles), extrait des Comptes rendus du $2^{e}$ Congrès international de philosophie, Genève, H. Kündig, [1904].

44. O. de Serres, Le theatre, op. cit., p. 87.

45. Jean Vienot, Histoire de la Réforme française, Paris, Fisherbacher, 1926-1934, p. 109; JeanJacques Boucher, Le dictionnaire de la soie..., Paris, ed. Lanore, 2014, p. 246.

46. Peut-être parce qu'une fois les œufs acquis et couvés, l'élevage des vers était confié à des paysans.

47. Aussi au XIV siècle, Le calendrier d'Ibn alBannâ de Marrakech (1256-1321 J. -C.), Renaud H.P.J. (trad., éd.), Institut des hautes études marocaines, t. 34, Paris, Larose, 1948.

48. David Scott Wilson-OKamura, Virgil in the Renaissance, Cambridge, Cambridge University Press, 2010.

49. Ludovico Lazzarelli, Il Bombyx, Rome, E. Silber, 1495; Pier Francesco Giustulo DA Spoleto, De sere seu setivomis animalibus, Rome,
G. Mazzocchi, 1500 et 1510; Marco Girolamo VidA,

De bombycum cura et usu, Rome, s. n., 1527.

50. Alessandro Tesauro, Della Sereide, Turin, Niccolò Bevilacqua, 1585.

51. À Tours, M. Sifleau; Henri Clouzot, « La sériciculture de Béroalde de Verville ", Revue du seizième siècle, 1915, t. 3, Paris, Champion, p. 281-286.

52. Brescia, D. Turlino, 1564 (11 feuillets, 56 p.)

53. Le Dieci giornate della vera agricoltura e piaceri della villa di M. Agostin Gallo, in dialogo, Vinegia, D. Sarri, 1565.

54. Les noms que l'on donne aux vers à soie dans différentes régions d'Italie.

55. Le vinti giornate dell'agricoltura t de pacieri della villa di M. Agostino Gallo..., Venise, G. Percaccino, 1569.

56. Giovanni Andrea Consuccio, Il vermicello dalla seta del Corsuccio da Sascorbaro nuovamente venuto in luce, Rimini, Gio Simbeni, 1581; il comprend trois livres dont seul le premier (68 p.) traite du ver à soie.

57. Ibid., p. 39, " se vede per chiara esperianza »; il s'agit de la première mue des vers et de l'affaiblissement qui s'ensuit accompagné de « una certa malinconia ».

58. Sur le développement de la production en Italie du Nord, Luca MoLÀ, The silk industry of Renaissance Venice, Baltimore, Johns Opkins University Press, 2000.

59. Charles Estienne, L'agriculture et maison rustique., augmentée par M. Jean Liebaut, Paris, Jacques Du Puys, 1570.

60. L'agricoltura et casa di villa di Carlo Stefano gentil'huomo francese, nuouamente tradotta da caualiere Hercole Cato..., Turin, Ratterij, 1583, p. 194 : « le Donne, che disegnano d'allevar cavalieri da seta, non devono aspettare nè beltà, nè quantità di more de suoi mori usando la foglia a nutrire cavalieri ».

61. Par exemple, Abu Zacaria Ebn el Awam de Séville, Le livre de l'agriculture, op. cit.

62. G. de LAs CAsas, Arte nuevo para criar seda..., Grenade, R. Rabut, 1581; Gabriel Alonso de Herrera, Agricoltura general... y los demas autores que hasta agora han escrito desta materia, cuyos nombres, y tratados van à la buelta desta hoja, Madrid, A. Martin, 1620, p. 209-224.

63. Woodrow W. BoraH, «El origen de la sericultura en la Mixteca Alta ", Historia mexicana, Collegio de Mexico, vol. 13, $\mathrm{n}^{\circ} 1$, juillet-septembre 1963, p. 1-17.

64. G. de Las Casas, Arte nuevo para criar seda..., op. cit., prologue, fol. $209 \mathrm{v}^{\circ}$.

65. Ainsi Léonard Fuchs qui, dans son Historia stirpium commentarii insignes..., Bâle, Isingriniana, 1542, p. 521-524, présente le mûrier et décrit la chronologie des mues du ver à soie; trad. française 
Histoire des plantes..., Lyon, G. Rouille, 1558, p. 362364 ; trad. espagnole par Joan JAVARA publiée à Anvers en 1557.

66. Ce qui est tout à fait conforme aux écrits de catholiques traitant des techniques durant la Contre Réforme.

67. Dans le Château de l'âme ou livre des demeures.

68. Christian ZANIER, « La fabrication de la soie : un domaine réservé aux femmes ", Travail, genre et sociétés, 2/2007, $\mathrm{n}^{\circ} 18$, p. 111-130.

69. A. Gallo, Le Dieci, trad. fr., op. cit., p. 199.

70. O. de SERRES, Le theatre, op. cit., p. 741.

71. M. VIDA, De bombycum, op. cit., p. 331, p. 71 de la trad. fr.
72. G. A. CoRsuccio, Il vermicello, op. cit., p. 18.

73. A. Gallo, Le Dieci, op. cit., Belleforest, 1571, épitre.

74. G. de Las Casas, Arte nuevo para criar seda, op. cit., fol. 210.

75. Ibid., fol. 230.

76. O. de SERRES, Le theatre, op. cit., p. 759.

77. Ibid., p. 760.

78. Ibid., p. 776.

79. Max WeBER, L'éthique protestante et l'esprit du capitalisme, Paris, Gallimard, 2004. 